\title{
Uncertainty Evaluation of Water Inrush in Karst Tunnels Based on Epistemic Uncertainty with Possibility Theory
}

\author{
Yiqing Hao $\mathbb{D},,^{1,2}$ Hao Lu $\mathbb{D},{ }^{3}$ Yehui Shi, ${ }^{4}$ Hao Geng $\mathbb{D},{ }^{3}$ Xi J, ${ }^{5}$ and Shufang Feng ${ }^{6}$ \\ ${ }^{1}$ Joint War College, National Defense University of PLA, Beijing 100091, China \\ ${ }^{2}$ High-tech Institute, Qingzhou, Shandong 262500, China \\ ${ }^{3}$ State Key Laboratory of Disaster Prevention and Mitigation of Explosion and Impact, Army Engineering University of PLA, \\ Nanjing 210007, China \\ ${ }^{4}$ Jiangsu Nanjing Geo-Engineering Investigation Institute, Nanjing 210000, China \\ ${ }^{5}$ Rocket Army Engineering Design and Research Institute, Beijing 100091, China \\ ${ }^{6}$ The No. $32395^{\text {th }}$ Troop of PLA, Shenyang 110000, China
}

Correspondence should be addressed to Hao Lu; lh829829@163.com

Received 19 January 2020; Revised 4 May 2020; Accepted 4 June 2020; Published 6 August 2020

Academic Editor: Anna M. Gil-Lafuente

Copyright $\odot 2020$ Yiqing Hao et al. This is an open access article distributed under the Creative Commons Attribution License, which permits unrestricted use, distribution, and reproduction in any medium, provided the original work is properly cited.

\begin{abstract}
In the risk assessment of water inrush in karst tunnel, it is most important to provide an available theoretical model for qualifying the epistemic uncertainties due to a lack of knowledge and information. Firstly, a mechanical model dependent on geology is introduced associating with four parameters, i.e., the elastic modulus $(E)$, the Poisson ratio $(\mu)$, the water differential pressure $(q)$, and the tunnel radius $(a)$. Then, a mathematical model representing epistemic uncertainty is represented with probability theory and possibility theory. The methodology was computerized to calculate the distribution of the margin and uncertainty and then to determine the ratio of "margin/uncertainty." Analyses involving possibility theory and possibility theory are illustrated with the same engineering example used in the presentation indicated above to illustrate the use of probability to represent aleatory and epistemic uncertainty in QMU analyses. The comparison between the uses of possibility theory and probability theory for the representation of aleatory and epistemic uncertainty indicates that the possibility is not only has a better mathematical structure than probability theory but also has some challenges.
\end{abstract}

\section{Introduction}

Risk and uncertainty are very important factors for decisionmaking [1]. Therefore, there is considerable research assessing the risk of water inrush. In general, quantitative analyses of water inrush occurring in tunnels during constructing are performed using mathematical and mechanical models that provide a representation of water inrush in tunnels based on a number of hypotheses and parameters. The model may be stochastic (e.g., fuzzy comprehensive evaluation, analytic hierarchy process, and attribution synthetic evaluation system) [2-4] or deterministic (e.g., mechanical model of outburst prevention layer) [5-7].

Stochastic models of water inrush risk are generally associated with qualitative and semiquantitative analysis.
Several indexes related to geology or construction may be considered in these models, and the weight coefficient is calculated through experience of the experts. For example, the attribution mathematics model is based on influencing factors and attribution measures calculated through a function derived from experience of the experts or data [2]. Fault tree analysis relies on approaches including extracting information from databases, solving stochastic differential equations, or relying on expert judgment to compute probabilities of basic events [3]. A fuzzy comprehensive evaluation system is another method constructed to evaluate risk using fuzzy mathematics theory [4], indices are determined based on the principles, and the weight of every index is distributed using analytic hierarchy process (AHP) or expertise. Based on the karst distribution pattern and the 
karst water inrush resisting rock strata, the assessment model for the karst water inrush risks is established [5]. Due to the great difference among influencing factors under different hydrogeological conditions, the risk prediction of water inrush of karst tunnels based on the BP neural network is adopted [6].

The models mentioned above are based on indices, weight coefficient, expert judgment, and so on, which are subjective parameters. The deterministic models overcome the aforementioned limitations, such as the mechanical model of the outburst prevention layer [7]. Using several variables, an analytical solution to assess the ground water inflow rate into a tunnel is presented using a mathematical derivation that considers excavation-induced rock permeability reduction in the vicinity of a tunnel based on the hydromechanical coupling effect [8].Deterministic models may consider influencing parameters such as water pressure and hydraulic conductivity $[9,10]$.

However, in practice, water inrush risk in tunnels cannot be characterized via analysis because the knowledge of the underlying phenomena for the water inrush is limited. This leads to uncertainties in the analysis, which cannot be described or foreseen due to intrinsic variability of water inrush itself or due to the lack of knowledge and information. Furthermore, this leads in practice to uncertainty on both values of the model parameters and on the hypotheses, which support the model structure. Such uncertainties propagate within the model and causes variability in its output, for example, when many values are plausible for a parameter, the output of the model is different. The quantification and characterization of the resulting output uncertainty is an important issue when the model of water inrush is used to guide decision-making. This topic is discussed as the uncertainty analysis of water inrush.

This paper aims at quantifying margins and uncertainties derived from uncertainties in the analysis input. We illustrate the ideas of uncertainty analysis by assuming that the deterministic model of water inrush in a tunnel is described as $f(X)$, which depends on the input quantities $X=\left\{x_{1}, x_{2}, \ldots\right.$, $x_{n}$ \} and on the function $f$; the quantity of interest $Y$ ( $Y$ is defined by the minimum safe thickness) is computed by using the deterministic model $Y=f(X)$. Therefore, the uncertainty analysis of $Y$ is described as a representation of $X$ and propagation through function $f$. Typically, the uncertainty about $X$ and $f$ are treated separately. The representation of $X$ has been developed using the probability theory in another article [11] and using the mechanical function $f$. If the representation of $X$ is obtained using different methods, the propagation is different under different conditions for the same function $f$.

There are two types of uncertainty in risk assessment: aleatory and epistemic uncertainty, which can be distinguished easily [12]. Aleatory uncertainty is related to the material properties of a component or system, which refers to the phenomena occurring in a random way. Thus, the probability method offers a sound and efficient approach to describe such uncertainty. Epistemic uncertainty is derived from the lack of knowledge about the properties or the behavior of the systems, which manifests in the representation of system behavior, in terms of both uncertainties of the hypotheses assumed and parameter uncertainty in fixed but poorly known values.

The representation of uncertainty of water inrush risk is provided in another paper. In the probabilistic approach, uncertainties are represented by probability associated with margins. However, it is challenged under the conditions of limited or lacking knowledge because a specific probability assignment is not provided due to the lack of knowledge and limited information. Furthermore, in a decision-making context, a probability assessment based on subjective judgment may not be satisfactory. This is related to a number of research studies in the other fields, which has led to the development of alternative representations [13]. Two representations may be suitable for water inrush risk assessment: possibility theory and evidence theory.

As indicated in the discussion of [14], there are also some questions for the use of possibility theory in the risk analyses of water inrush in the karst tunnel. These questions include (1) the transition of disseminated information into possibility theory structure, (2) the accumulation of information for different sources into suitable possibility theory structure [15], (3) the propagation of multidimensional possibility spaces [15-18], and (4) the communication of intuitions and views drawn from risk analysis of water inrush in the karst tunnel that uses possibility theory to characterize uncertainty $[19,20]$. Although there are some challenges existing on the use of possibility theory for representation of epistemic uncertainty, it is also important to notice that similar challenges exist on the use of probability theory of representation of epistemic uncertainty.

The remainder of this manuscript is organized as follows. In Section 2, the mechanical model of water inrush in the karst tunnel (i.e., the deterministic model or function) is illustrated, followed by description of the model of water inrush, the use of possibility in the representation of epistemic uncertainty, and the QMU analysis only that involves only epistemic uncertainty in Section 3. Then, the differences among the representations of probability theory, possibility theory, and evidence theory are discussed in Section 4. In Section 5, a summary is provided to conclude the paper.

\section{Mechanical Model of Water Inrush in a Tunnel Used for Illustration}

The mechanical model used in this paper is based on geology with no defects, defined by the model of outburst prevention layer in a tunnel face, and measured with the safe thickness of the outburst prevention layer. In particular, the behavior of the water inrush is described by the equation [21]

$$
\mathbf{l}=\sqrt[3]{\frac{3 \mathbf{a}^{3}(1-\boldsymbol{\mu}) \mathbf{q}}{40 \mathbf{E}}}
$$

where $\mathbf{E}$ is elastic modulus, $\mu$ is Poisson ratio, $\mathbf{q}$ is water differential pressure, and $\mathbf{l}$ is the tunnel radius.

The vector $\mathrm{e}_{\mathrm{M}}$ of epistemic uncertain input is described as 


$$
e_{M}=\left[e_{M 1}, e_{M 2}, e_{M 3}, e_{M 4}\right]=[E, \mu, a, q] .
$$

It is clear and convenient that $e_{M 1}, e_{M 2}, e_{M 3}$, and $e_{M 4}$ are used instead of $E, \mu, a$, and $q$ to note the element of $e_{M}$. Furthermore, the risk of water inrush is assumed to operate under conditions on an unstated vector of aleatory uncertainty, which is considered a fixed realization. In this context, one might correspond to specified conditions for the system. Thus, the representation of the safe thickness $\mathbf{l}$ is described as the notation of $l\left(a, e_{M}\right)$ with the incorporation of $a$ and $e_{M}$. The aleatory uncertainty in this context does not exist, and therefore, only epistemic uncertainty is considered.

Uncertainty structures only with epistemic uncertainty are specified for $E, \mu, a$, and $q$ based on possibility and evidence theory. Then, the resultant uncertainty structures for $\mathbf{l}$ and related quantities are described.

The appropriate or possible values for $E, \mu, a$, and $q$ are assumed to belong to the intervals, respectively, as shown in equations (3)-(6):

$$
\begin{aligned}
& \varepsilon M_{1}=\left\{E: E_{m n} \leq E \leq E_{m x}\right\}=\{E: 1.8 \mathrm{GPa} \leq E \leq 3.2 \mathrm{GPa}\}, \\
& \varepsilon M_{2}=\left\{\mu: \mu_{m n} \leq \mu \leq \mu_{m x}\right\}=\{\mu: 0.22 \leq \mu \leq 0.28\}, \\
& \varepsilon M_{3}=\left\{a: a_{m n} \leq a \leq a_{m x}\right\}=\{a: 4.7 \mathrm{~m} \leq a \leq 5.3 \mathrm{~m}\}, \\
& \varepsilon M_{4}=\left\{q: q_{m n} \leq q \leq q_{m x}\right\}=\{q: 2.2 \mathrm{GPa} \leq q \leq 3.8 \mathrm{GPa}\} .
\end{aligned}
$$

The only information about the appropriate values for $E, \mu, a$, and $q$ is assumed to be known as described by equations (3)-(6); no more information is provided.

\section{QMU with Epistemic Uncertainty: Characterization with Possibility Theory}

3.1. Uncertainty Representation Using Possibility Theory. Possibility theory [22] is based on the specification of a pair $(X, \pi)$, where (i) $X$ is the set of possible values for $x$ and (ii) $\pi$ is a function defined such that $0 \leq \pi_{X}(x) \leq 1$ for $x \in X$ and $\sup \{\pi(x): x \in X\}=1$. The function can be seen as a measure of the amount of "confidence" assigned to each element of $x$ and is referred to as the possibility distribution function for $x$. The possibility distribution $\pi_{X}(x)$ reflects the degree of similarity between $x$ and the true value, and it indicates the distance between $x$ and the true value. Thus, in some cases, this may be determined objectively using a defined measurement procedure [23]. In other cases, it may be based on the subjective judgment of an expert or analyst. Therefore, $\pi_{X}(x)=0$ means that water inrush is considered as an impossible accident, while $\pi_{X}(x)=1$ means water inrush is possible or just unsurprising, normal, or usual [24]. However, it is much weaker than when the probability is equal to 1 .

Rationally, epistemic uncertainty can be described using possibility distribution because a possibility distribution defines a family of probability distributions [25-27]. Therefore, the probability distributions are bounded above by the so-called possibility function and below by so-called necessity function.

3.2. Uncertainty Representation for a Variable. Possibility theory refers to two measures: possibility and necessity. In particular, possibility and necessity for a subset $A$ of $X$ are defined by

$$
\begin{aligned}
& \Pi(A)=\sup _{x \in X} \pi_{X}(x), \\
& N(A)=1-\Pi(\bar{A})=\inf _{x \notin X}\left(1-\pi_{X}(x)\right) .
\end{aligned}
$$

In keeping with the possibility distribution function, $\Pi(A)$ can supply a measure of the existence of information that does not oppose the proposition that $A$ contains the appropriate value for $X$, and $N(A)$ can supply a measure of the existence of uncontradicted information that supports the proposition that $A$ contains the appropriate value for $X$.

In possibility theory, relationships are satisfied as [28]

$$
\begin{gathered}
\Pi(A)+N(\bar{A})=\Pi(\bar{A})+N(A)=1, \\
N(A) \leq \Pi(A), \\
\Pi(A)+\Pi(\bar{A}) \geq 1, \\
N(\bar{A})+N(A) \leq 1, \\
\max \{\Pi(A), \Pi(\bar{A})\}=1, \\
\min \{N(A), N(\bar{A})\}=0, \\
\Pi(A) \leq 1 \Rightarrow N(A)=0, \\
N(A) \geq 0 \Rightarrow \Pi(A)=1 .
\end{gathered}
$$

For possibility theory, four subintervals are considered for each of the intervals $\varepsilon M_{i}, i=1,2,3$, and 4 , as described by equations (3)-(6):

$$
\begin{aligned}
\mathrm{E}_{i 1} & =\left[c, b-\frac{(b-c)}{4}\right], \\
\varepsilon_{i 2} & =\left[c+\frac{(b-c)}{4}, b\right], \\
\varepsilon_{i 3} & =\left[c+\frac{(b-c)}{8}, b-\frac{3(b-c)}{8}\right], \\
\varepsilon_{i 4} & =\left[c+\frac{3(b-c)}{8}, b-\frac{(b-c)}{8}\right],
\end{aligned}
$$

where $[\mathrm{c}, \mathrm{b}]$ corresponds to $\left[E_{m n}, E_{m x}\right],\left[\mu_{m n}, \mu_{m x}\right]$, $\left[a_{m n}, a_{m x}\right]$, and $\left[q_{m n}, q_{m x}\right]$ for $i=1,2,3$, and 4 , respectively. In turn, the possibility distribution function $\pi_{E M, i}\left(e_{M i}\right)$ is given by 


$$
\begin{aligned}
\pi_{E M, i}\left(e_{M i}\right) & =\sum_{j=1}^{4} \frac{\delta_{i j}\left(e_{M i}\right)}{4}, \\
\delta_{i j}\left(e_{M i}\right) & = \begin{cases}1, & \text { if } e_{M i} \in \varepsilon_{i j}, \\
0, & \text { otherwise. }\end{cases}
\end{aligned}
$$

According to equations (3)-(6) and (16)-(21), the possibility distributions $\pi_{E M, i}\left(e_{M i}\right), i=1,2,3,4$, for $E, \mu, a$, and $q$ are described, as shown in Figure 1.

3.3. Uncertainty Propagation. According to the uncertainty propagation framework suggested in the previous section, a model is assumed whose output is a function (as shown in equation (1)), concerning $n$ uncertain variables $X_{j}, j=1,2, \ldots, 4$, (i.e., $X_{1}=E, X_{2}=\mu, X_{3}=a$, and $X_{4}=q$ ) which are "possibilistic." This means that uncertainty is described by possibility distributions (or fuzzy distributions) [29] $\pi_{E}\left(x_{1}\right), \pi_{\mu}\left(x_{2}\right), \pi_{a}\left(x_{j}\right)$, and $\pi_{q}\left(x_{n}\right)$. In such a case, the propagation of uncertainty can be defined by fuzzy interval analysis (FIA) in possibility theory [30]. In summary, it can be seen that convolutions defining fuzzy arithmetic essentially reduce to interval arithmetic repeated many times, once for each level of possibility; however, unlike interval analysis, fuzzy arithmetic [31] provides a possibility distribution rather than a simple range.

In particular, the steps of the uncertainty propagation procedure are operated by FIA as follows [32]:

(1) Suppose $\alpha=0$.

(2) Use the line $\alpha$ to cut the possibility distributions $\pi_{E}\left(x_{1}\right), \pi_{\mu}\left(x_{2}\right), \pi_{a}\left(x_{3}\right)$, and $\pi_{q}\left(x_{4}\right)$ separately. Then, the $\alpha$-cuts $A_{\alpha}^{E}, A_{\alpha}^{\mu}, A_{\alpha}^{a}$, and $A_{\alpha}^{q}$ of the "possibilistic" variables $X_{j}, j=1,2,3,4$, are selected, and thus, the interval of possible values $\underline{x}_{j, \alpha}, \bar{x}_{j, \alpha}, j=1,2,3,4$, as shown in Figure 2.

(3) According to the interval of possible values $\underline{x}_{j, \alpha}, \bar{x}_{j, \alpha}, j=1,2,3,4, \quad$ and $\quad l=Y=f(X)=$ $f(E, \mu, a, q) . X_{j}$ is a random sample $e_{M i}(i=1,2, \ldots$, $n S E=1000)$; then, compute the values of $Y$, and select the smallest and largest values of $Y$, which are denoted by $y_{\alpha}$ and $\bar{y}_{\alpha}$. Similarly, the variable range $X_{j} \quad$ is selected within the intervals $\underline{x}_{j, \alpha}, \bar{x}_{j, \alpha}, j=1,2,3,4$ :

$$
\begin{aligned}
& \underline{y}_{\alpha}=\inf _{j, X_{j} \in\left|\underline{x}_{j, \alpha}, \bar{x}_{j, \alpha}\right|} f\left(X_{1}, X_{2}, X_{3}, X_{4}\right), \\
& \bar{y}_{\alpha}=\sup _{j, X_{j} \in\left|\underline{x}_{j, \alpha}, \bar{x}_{j, \alpha}\right|} f\left(X_{1}, X_{2}, X_{3}, X_{4}\right) .
\end{aligned}
$$

(4) Thus, the values $y_{\alpha}$ and $\bar{y}_{\alpha}$ calculated above in step 3 are considered as the lower and upper limits (i.e., the smallest and largest values) of the $\alpha$-cuts $A_{\alpha}^{Y}$ of $y$.

(5) Next, if $\alpha<1$, set $\alpha=\alpha+\Delta \alpha$ (e.g., $\Delta \alpha=0.001$ ) and return to step 2, above; otherwise, stop the algorithm.
Then, the possibility distribution $\pi_{Y}(Y)$ of $Y=f\left(X_{1}, X_{2}, X_{3}, X_{4}\right)$ is provided across the accumulation of the values $y_{\alpha}$ and $\bar{y}_{\alpha}$ from each $\alpha$-cuts (notice that since $\Delta \alpha=0.001$, then $N_{\alpha}=(q+1)=$ $1 /(\Delta \alpha+1)=1 /(0.001+1)=1001$ values of are considered in the computational procedure, i.e., $N_{\alpha}=1001 \alpha$-cuts of the possibility distributions $\pi_{E}\left(x_{1}\right), \pi_{\mu}\left(x_{2}\right), \pi_{a}\left(x_{3}\right)$, and $\pi_{q}\left(x_{4}\right)$ are selected. Thus, the possibility distribution $\pi_{Y}(Y)$ of $Y=f\left(X_{1}, X_{2}, X_{3}, X_{4}\right)$ is constructed as the collection of its $N_{\alpha}=(q+1)=1 /(\Delta \alpha+1)=1 /$ $(0.001+1)=1001 \alpha$-cuts intervals $\left.\left|\underline{Y}_{\alpha}, \bar{Y}_{\alpha}\right|\right)$.

According to equations (1), (3)-(6), and (17)-(22) and Figure 1 , the possibility distributions $\pi_{l}$ for 1 can be computed based on the propagation procedure, as shown in Figure 2.

It is importance noting that performing an interval analysis on $\alpha$-cuts assumes total dependence between the parameters involving epistemic uncertainty. In fact, this propagation procedure implies potent dependence between the sources of information (e.g., the experts or observers) that provide the input possibility distributions because the same confidence level $(1-\alpha)$ is selected to construct the $\alpha$-cuts for all uncertain variables [33].

3.4. Epistemic Uncertainty without a Specified Bound. As discussed previously, the possible values for $l\left(a, e_{M}\right)$ are considered, which are

$$
l=l\left(a, e_{M}\right): e_{M i}=[E, \mu, a, q],
$$

where $e_{M}$ is considered a fixed realization associated with aleatory uncertainty. $l$ has an uncertainty form deriving from the uncertainties of $\varepsilon M$. Then, the uncertainty of $l\left(a, e_{M}\right)$ can be characterized by an interval (i.e., $[\Pi(A), N(A)])$.

According to equations (6) and (7) and the possibility distribution $\pi_{l}$ shown in Figure 2, the cumulative distributions and complementary cumulative distributions of possibility values for $l\left(a, e_{M}\right)$ are described in Figures 3 and 4 , respectively.

The cumulative distributions and complementary cumulative distributions have step sizes of 0.25 because the possibility distribution function for $l\left(a, e_{M}\right)$ has step sizes of 0.25 (Figure 2), which is computed through the possibility distributions $\pi_{E M, i}\left(e_{M i}\right), i=1,2,3,4$, and uncertainty propagation procedure (equations (6) and (7) and (17)-(24)).

Taking the possible value of 1.8 for $l\left(a, e_{M}\right)$ as an example (dash lines in Figures 5 and 6), all information known about the value of 1.8 is involved in the interval $[\inf (l), \sup (l)]=[1.626,2.586]$, which indicates the possible values for $l\left(a, e_{M}\right)$. In the interval analysis results, there is nothing else known about 1.8 or the confidence levels of the other potential values for $l\left(a, e_{M}\right)$ near the location of 1.8. That is to say, no more information is provided for giving to the possibility of whether the inequality $l\left(a, e_{M}\right) \leq 1.8$ is true or the inequality $l\left(a, e_{M}\right) \geq 1.8$ is true. 


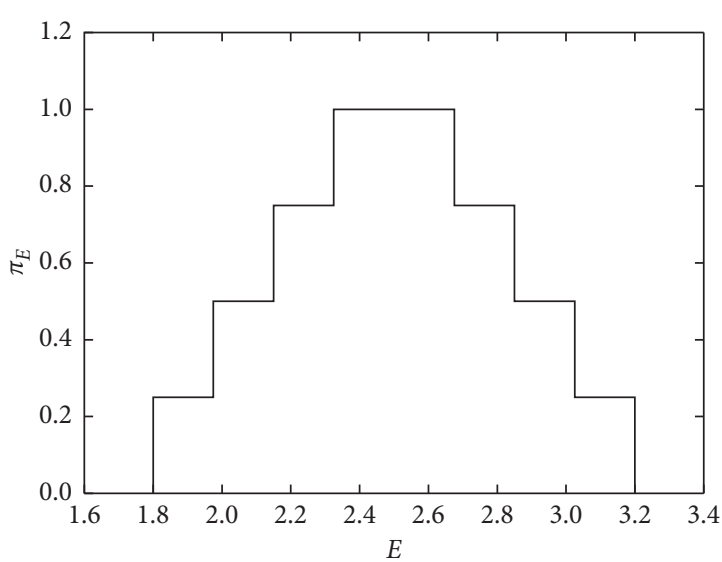

(a)

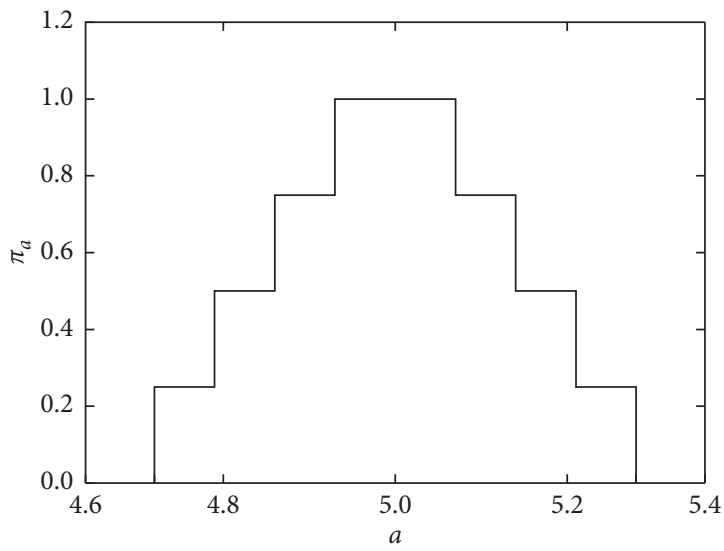

(c)

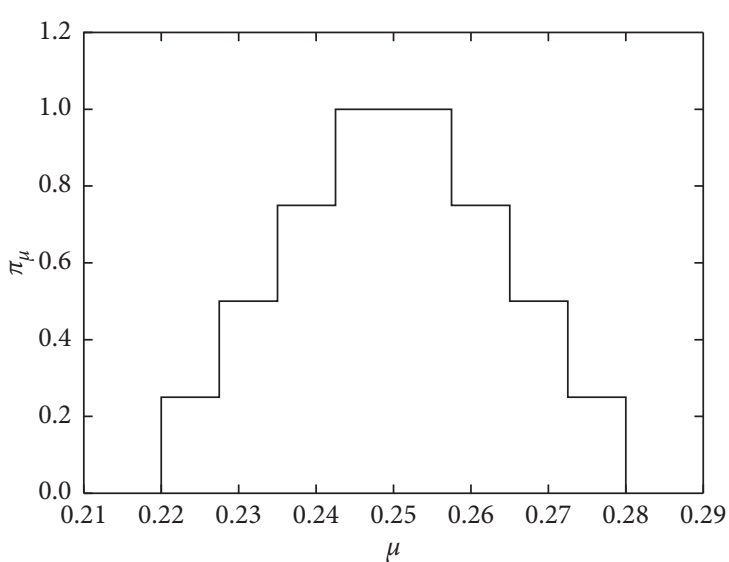

(b)

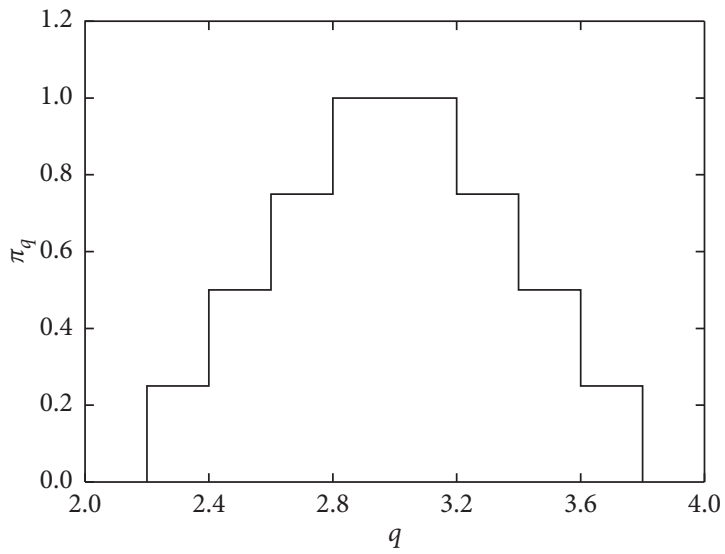

(d)

Figure 1: Possibility distributions: (a) $\pi_{E}$ for $E$ (b) $\pi_{\mu}$ for $\mu$, (c) $\pi_{a}$ for $a$, and (d) $\pi_{q}$ for $q$. They should be listed as (a) possibility distribution $\pi_{E M, i}$ for $E$; (b) possibility distribution $\pi_{E M, i}$ for $\mu$; (c) possibility distribution $\pi_{E M, i}$ for $a$; and (d) possibility distribution $\pi_{E M, i}$ for $q$.

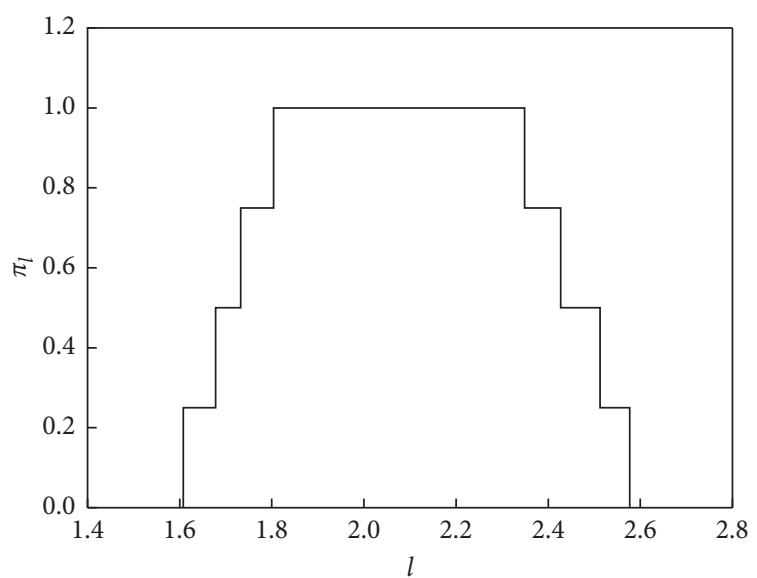

FIgURe 2: Possibility distributions $\pi_{l}$ of output 1 obtained by FIA with $\alpha$-cuts intervals.

As shown in Figure 5, more information about the possible value of 1.8 for $l\left(a, e_{M}\right)$ is now discussed. At the beginning, the condition that measures of credence exist for the potential values of $l\left(a, e_{M}\right)$ less than or equal to 1.8 is considered.
Suppose the measures of credence exist for a set, which is described as

$$
l_{1.8}=\{\bar{l}: \bar{l} \in l \text { and } \bar{l} \leq 1.8\} .
$$

Then, the measures can be described as equation (26) for possibility theory:

$$
\left[N_{l}\left(l_{1.8}\right), \Pi_{l}\left(l_{1.8}\right)\right]=[0.0,0.75] .
$$

Possibility and necessity, which are denoted by $N_{l}\left(l_{1.8}\right)$ and $\Pi_{l}\left(l_{1.8}\right)$, respectively, are two measures of credence combined with the set $l_{1.8}$ :

$$
0=N\left(l_{1.8}\right) \leq \Pi_{l}\left(l_{1.8}\right)=0.75,
$$

where $N_{l}\left(l_{1.8}\right)$ means a measure of presentation that does not contradict information supporting the proposition that the suitable value for $l\left(a, e_{M}\right)$ is contained in $l_{1.8}$, and $\Pi_{l}\left(l_{1.8}\right)$ means a measure of presentation for the information which does not refute the proposition that the suitable value for $l\left(a, e_{M}\right)$ is contained in $l_{1.8}$.

The condition that measures of the credence exist for potential values of $l\left(a, e_{M}\right)$ greater than 1.8 is now considered. Suppose the measures of credence exist for a set, which can be described as 


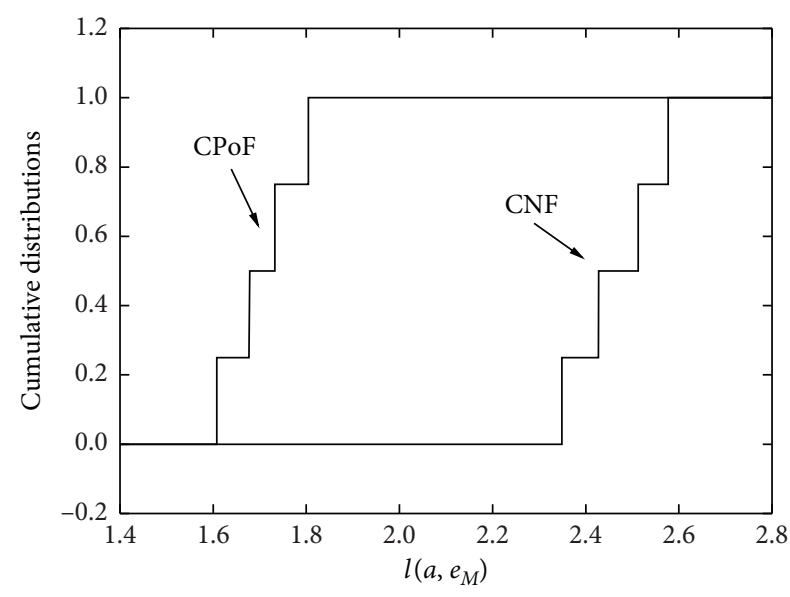

FIGURE 3: Cumulative distributions of uncertainty associated with $l\left(a, e_{M}\right)$ characterized by possibility summarized with $\mathrm{CPoF}$ and CNF.

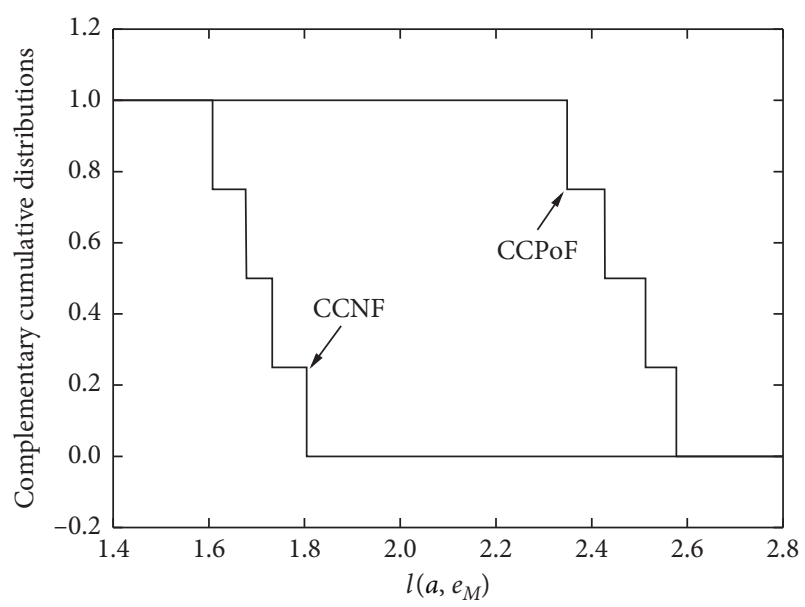

FIgURE 4: Cumulative distributions of uncertainty associated with $l\left(a, e_{M}\right)$ characterized by possibility summarized with CPoF and CNF.

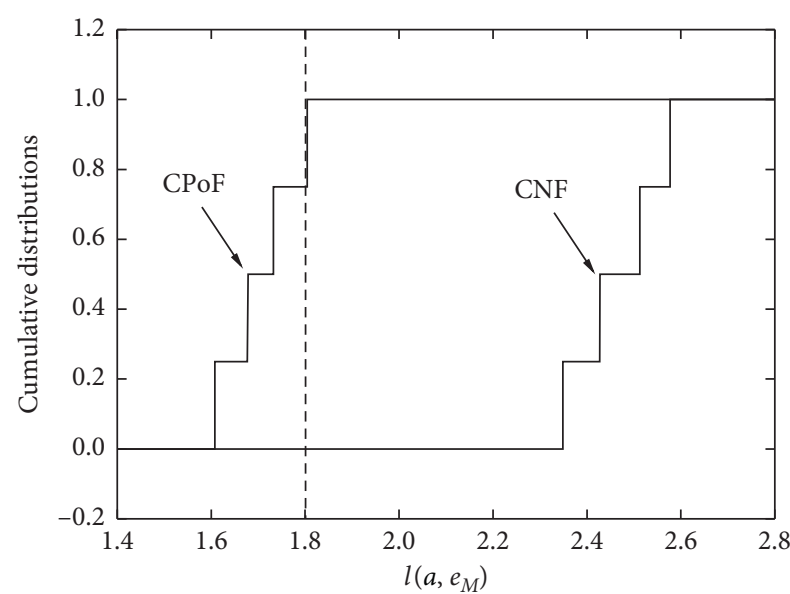

Figure 5: CPoF and CNF of uncertainty associated with $l\left(a, e_{M}\right)$ corresponding to the locations where the vertical line crossed.

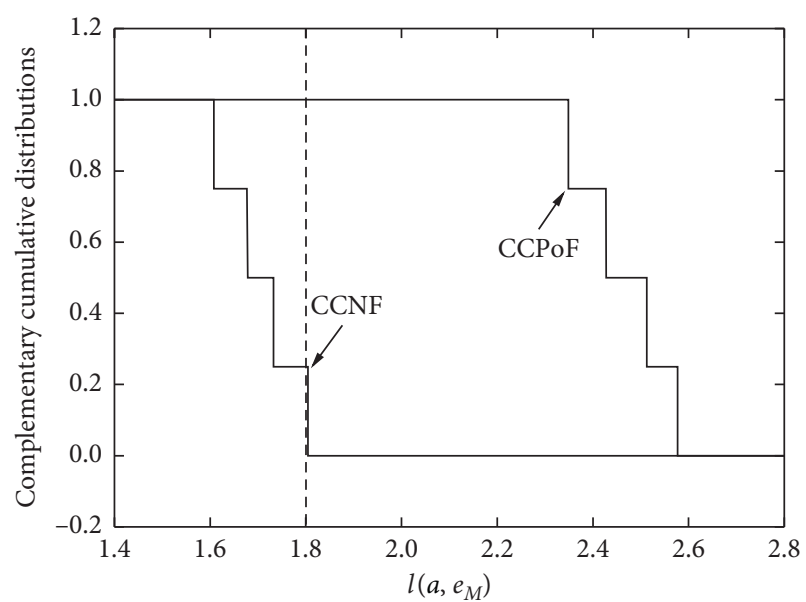

FIgURE 6: CCPoF and CCNF of uncertainty associated with $l\left(a, e_{M}\right)$ corresponding to the locations where the vertical line crossed.

$$
l_{1.8}^{c}=\{\bar{l}: \bar{l} \in l \text { and } \bar{l} \geq 1.8\} \text {. }
$$

Then, the measures can be described as equation (29) for possibility theory:

$$
\left[N_{l}\left(l_{1.8}^{c}\right), \Pi_{l}\left(l_{1.8}^{c}\right)\right]=[0.25,1.0] .
$$

As discussed above, $N_{l}\left(l_{1.8}^{c}\right)=0.25$ provides a measure of credence in the possibility theory, which is the presentation of no contradicted information supporting the proposition that the suitable value for $l\left(a, e_{M}\right)$ is contained in $l_{1.8}^{c}$. Likewise, $\Pi_{l}\left(l_{1.8}^{c}\right)=1.0$ provides a measure of the credence in the possibility theory, which is the presentation of information that does not refute the proposition that the suitable value for $l\left(a, e_{M}\right)$ is contained in $l_{1.8}^{c}$.

The relationships existing between measures of confidence for suitable values and measures of confidence for the complements of the suitable values are indicated as equations (31) and (32), holding as indicated in equation (8):

$$
\begin{aligned}
& 1=N_{l}\left(l_{1.8}\right)+\Pi_{l}\left(l_{1.8}^{c}\right)=0.0+1.0, \\
& 1=N_{l}\left(l_{1.8}^{c}\right)+\Pi_{l}\left(l_{1.8}\right)=0.25+0.75 .
\end{aligned}
$$

Extrarelationships are considered for possibility theory as indicated in equations (8)-(16). On the whole, it is more nuanced for the representation of epistemic uncertainty with possibility theory than with probability theory because possibility theory provides discrimination between information that does not contradict a proposal and information that supports a proposal.

3.5. Epistemic Uncertainty with a Specified Bound. QMU analysis with a fixed bound concerning the value for $l\left(a, e_{M}\right)$ is considered. Two types of bounds are considered, bounding $l\left(a, e_{M}\right)$ from below (e.g., $l_{b 1}=1.55$ and $l_{b 2}=1.75$, seen in Figure 7 ) and from above (e.g., $l_{b 3}=2.4$ and $l_{b 4}=2.6$, seen in Figure 8).

As shown in Figure 7, all possible values for $l\left(a, e_{M}\right)$ are above the bound $l_{b 1}=1.55$, but this is different for the bound 


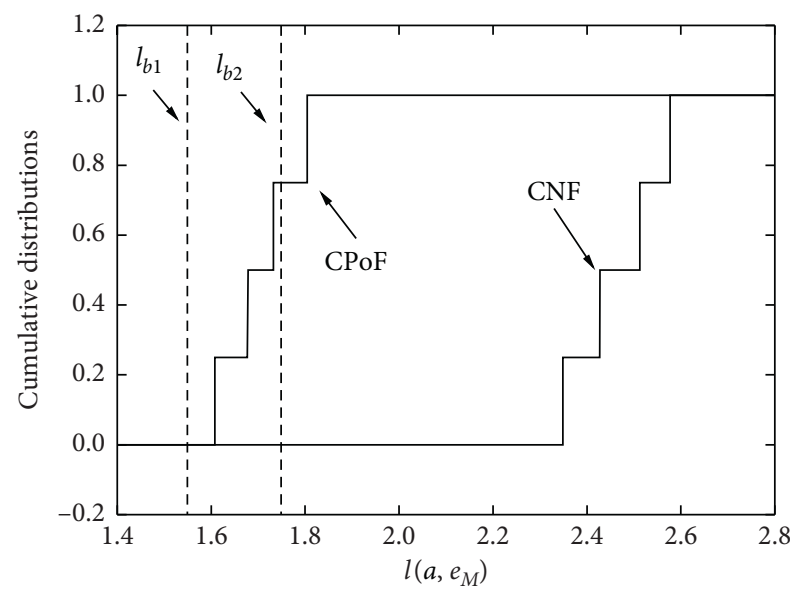

FIgURE 7: Bounding $l\left(a, e_{M}\right)$ from below: $l_{b 1}=1.55$ and $l_{b 2}=1.75$.

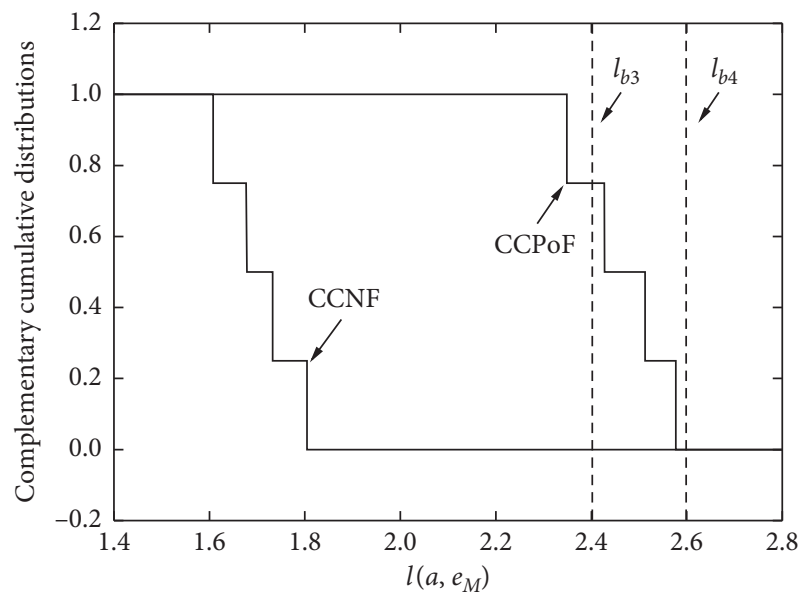

FIgURE 8: Bounding $l\left(\mathrm{a}, e_{M}\right)$ from above: $l_{b 3}=2.4$ and $l_{b 4}=2.6$.

$l_{b 2}=1.75$. The possibility and necessity below the bound $l_{b 2}=1.75$ are 0.75 and 0.0 , respectively.

Similarly, as shown in Figure 8, all possible values for $l\left(a, e_{M}\right)$ are below the bound $l_{b 4}=2.6$, but this is different for the bound $l_{b 3}=2.4$. The possibility and necessity exceeding the bound $l_{b 3}=2.4$ are 0.75 and 0.0 , respectively.

According to the cumulative distributions and complementary cumulative distributions of $l\left(a, e_{M}\right)$, the margins between $l\left(a, e_{M}\right)$ and bounds $l_{b k}, k=1,2,3$, and 4 , are defined as

$$
l_{m k}\left(a, e_{M}\right)= \begin{cases}l\left(a, e_{M}\right)-l_{b k}, & \text { for } k=1,2, \\ l_{b k}-l\left(a, e_{M}\right), & \text { for } k=3,4,\end{cases}
$$

where $l_{m k}\left(a, e_{M}\right) \geq 0$ means that a bound is satisfied and $l_{m k}\left(a, e_{M}\right)<0$ means that a bound is not satisfied. Therefore, it is good for a positive margin and bed for a negative margin. Because $l\left(a, e_{M}\right)$ is uncertain, the margins $l_{m k}\left(a, e_{M}\right), k=1,2,3,4$, are uncertain too. The uncertainty structure of $l_{m k}\left(a, e_{M}\right), k=1,2,3,4$, propagated by uncertainties assumed for $e_{M}$ (i.e., $E, \mu, a$, and $q$ ) is described as Figure 9. Furthermore, a complete QMU representation associated with a complete representation of margins $l_{m k}\left(a, e_{M}\right), k=1,2,3,4$, and uncertainty is described by the representation shown in Figure 9.

Sometimes, a QMU representation can be provided by the ratio of "margin/uncertainty," defined by equation (34) and depicted as Figure 10.

$$
l_{n k}\left(a, e_{M}\right)=\frac{l_{m k}\left(a, e_{M}\right)}{l_{b k}}= \begin{cases}\frac{\left[l\left(a, e_{M}\right)-l_{b k}\right]}{l_{b k}}, & \text { for } k=1,2, \\ \frac{\left[l_{b k}-l\left(a, e_{M}\right)\right]}{l_{b k}}, & \text { for } k=3,4 .\end{cases}
$$

The form of the representation described as the ratio of "margin/uncertainty" contains not only the margin but also the bounding value that some individuals may like. However, the form does not describe the actual size of the margin, and an important amount of information is lost because the margin is presented as a multiple of the bounding value.

According to discussions above, QMU representation with possibility theory is a good attempt to reduce the complicated analysis to a single number when the knowledge is limited.

\section{Relationship between Epistemic Uncertainty with Probability Theory and Possibility Theory}

As discussed above, the possibility distribution representing epistemic uncertainty can describe a family of probability distribution. The possibility function defines the boundary above the probability distributions and the necessity function defines the boundary below the probability distributions.

According to the previous section and the representation of epistemic uncertainty with probability theory discussed in [11], the differences in uncertainty associated with margins $l_{m k}\left(a, e_{M}\right), k=1,2,3,4$, between the possibility and probability theories are shown in Figure 11. Similarly, the differences in uncertainty connected with another denotation defined as normalized margins (i.e., the ratio of "margin/ uncertainty") can be described in Figure 12.

As shown in Figures 11 and 12, the possibility of margins is the probability bound above and the necessity is the probability bound below. Simultaneously, there are some differences in the representation of epistemic uncertainty characterized by the possibility and probability theories.

4.1. Possibility and Necessity Can Be Described as Probability Bounds. As shown in Figure 2, the possibility distribution can be viewed as a nested set of confidence intervals, denoted by $A_{\alpha}^{Y}=\left|\underline{y}_{\alpha}, \bar{y}_{\alpha}\right|=\left\{y, \pi_{Y}(Y) \geq \alpha\right\} . N\left(\left|\underline{y}_{\alpha}, \bar{y}_{\alpha}\right|\right)$ is the degree of certainty that the value of an uncertain variable is contained in $A_{\alpha}^{Y}$, while $\pi_{Y}(Y)$ is continuous. In particular, the quantity of the uncertainty is a most conservative estimate at the possibility level of $\alpha=0$ because the range of values is the widest, which means that the range would contain the true value in the interval. Similarly, the great optimism 


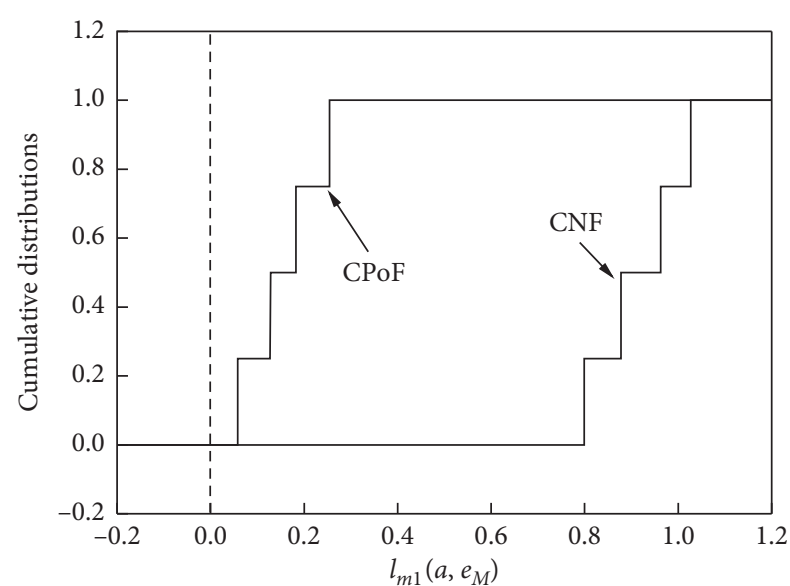

(a)

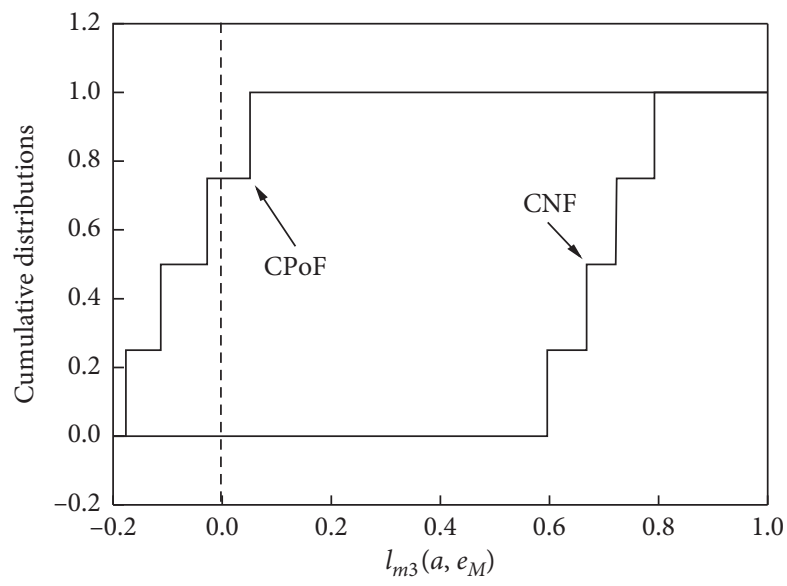

(c)

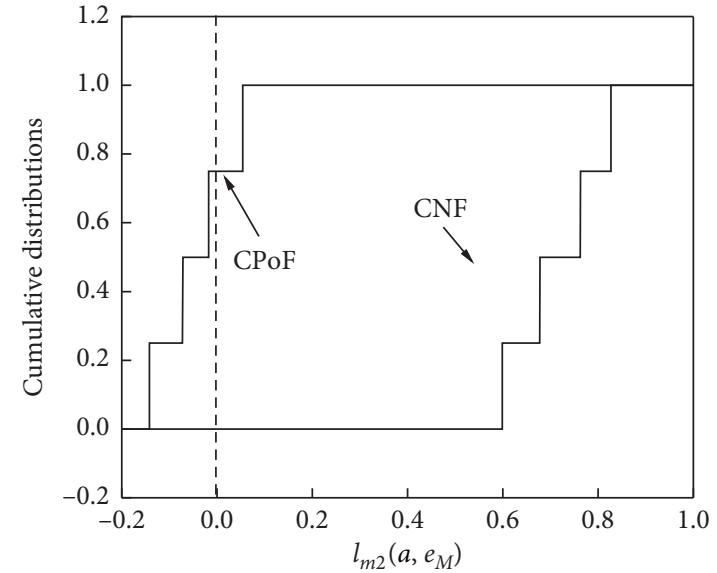

(b)

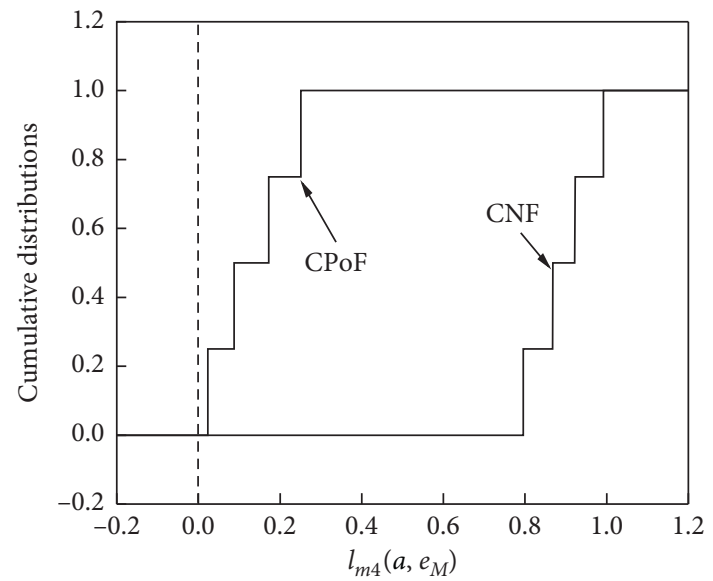

(d)

FIGURE 9: Uncertainty connected with margins $l_{m k}\left(\mathrm{a}, e_{M}\right), k=1,2,3,4$, defined in equation (32) characterized by possibility summarized with CPoF and CNF: (a) $l_{m 1}\left(a, e_{M}\right)$ for $l_{m 1}=1.55$, (b) $l_{m 2}\left(a, e_{M}\right)$ for $l_{n 2}=1.75$, (c) $l_{m 3}\left(a, e_{M}\right)$ for $l_{m 3}=2.4$, and (d) $l_{m 4}\left(a, e_{M}\right)$ for $l_{m 4}=2.6$.

corresponds to the possibility level of $\alpha=1$ because the range of values is the narrowest, which would be the best estimate of the uncertainty. If $0<\alpha<1$, the ranges of values are intermediately expressed by possibility.

Suppose a nested of intervals $A_{i}$ with degrees of necessity $N\left(A_{i}\right)$ containing values of uncertain variables, where $A_{i} \in A_{i+1}, i=1,2, \ldots, m-1$; then, the body of evidence $\left[\left(A_{1}, N\left(A_{1}\right)\right),\left(A_{2}, N\left(A_{2}\right)\right), \ldots,\left(A_{m}, N\left(A_{m}\right)\right)\right]$ is formed. The possibility distribution obeys

$$
\pi_{Y}(y)= \begin{cases}1, & \text { if } y \in\left(A_{i}\right), \\ \min _{i: y \notin A_{i}} 1-N\left(A_{i}\right), & \text { otherwise. }\end{cases}
$$

Therefore, the subjective probability $p_{Y}(A)$ is at least equal to $N(A)$. In particular, the $\alpha$-cut of the continuous possibility distribution can be described as

$$
p\left(\text { uncertain variable } \in\left|\underline{y}_{\alpha}, \bar{y}_{\alpha}\right|\right) \geq 1-\alpha,
$$

which is equivalent to the inequality

$$
p\left(\text { uncertain variable } \notin\left|\underline{y}_{\alpha}, \bar{y}_{\alpha}\right|\right) \leq \alpha \text {. }
$$

Thus, any necessity/possibility function can be interpreted as lower/upper probabilities from specific probability families (i.e., degrees of possibility can be described as upper probability bounds and degrees of necessity can be described as lower probability bounds).

Formally, $\pi_{Y}$ is denoted as a possibility distribution including necessity and possibility functions of $[N, \Pi]$. The probability family can be defined as

$$
\begin{aligned}
& \left\{p_{Y}, \forall \text { Ameasureable: } N(A)<p_{Y}(A)\right\} \text { and } \\
& \left\{p_{Y}, \forall A \text { measureable: } p_{Y}(A)<\Pi(A)\right\} .
\end{aligned}
$$

It also can be described as

$$
\begin{aligned}
& \sup _{p_{Y}} p_{Y}(A)=\Pi(A), \\
& \inf _{p_{Y}} p_{Y}(A)=N(A) .
\end{aligned}
$$

Thus, we know that $p_{Y} \in\left\lfloor\underline{p}_{Y}, \bar{p}_{Y}\right\rfloor, \underline{p}_{Y}=N$, and $\bar{p}_{Y}=\Pi$.

4.2. Qualitative Comparisons of Probability Theory and Possibility Theory. As discussed above, possibility and necessity 


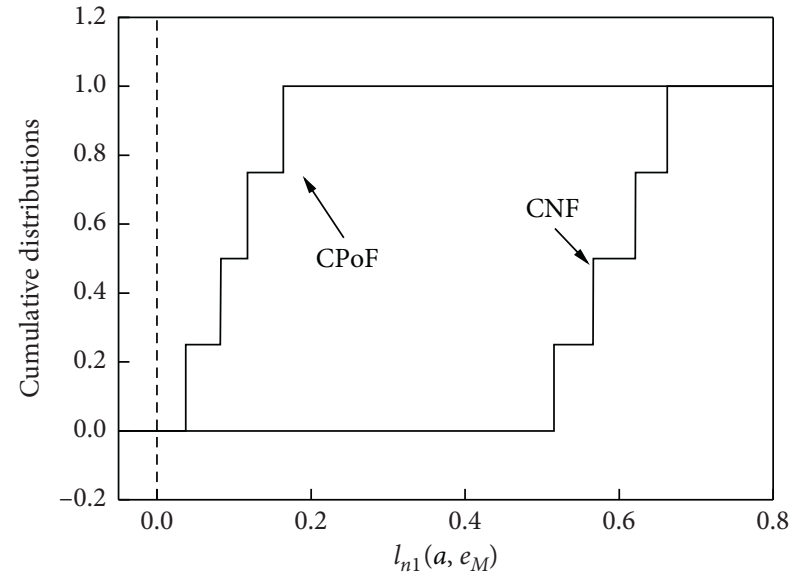

(a)

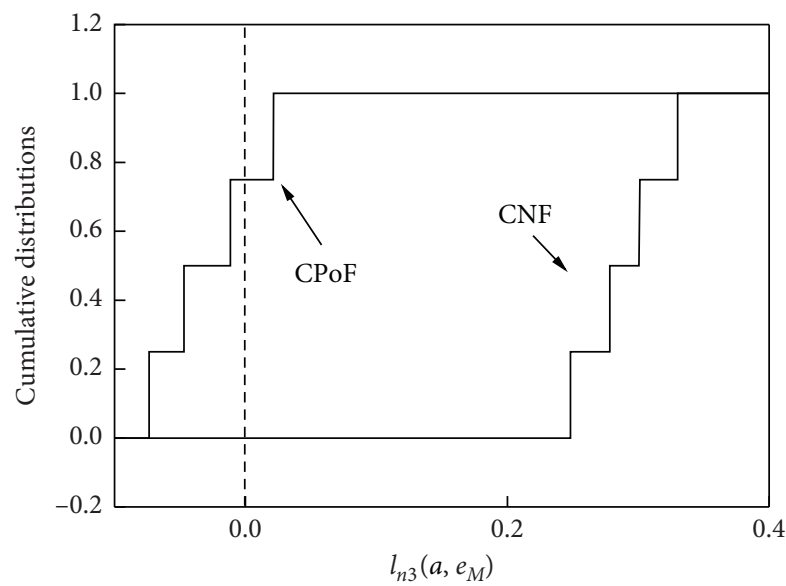

(c)

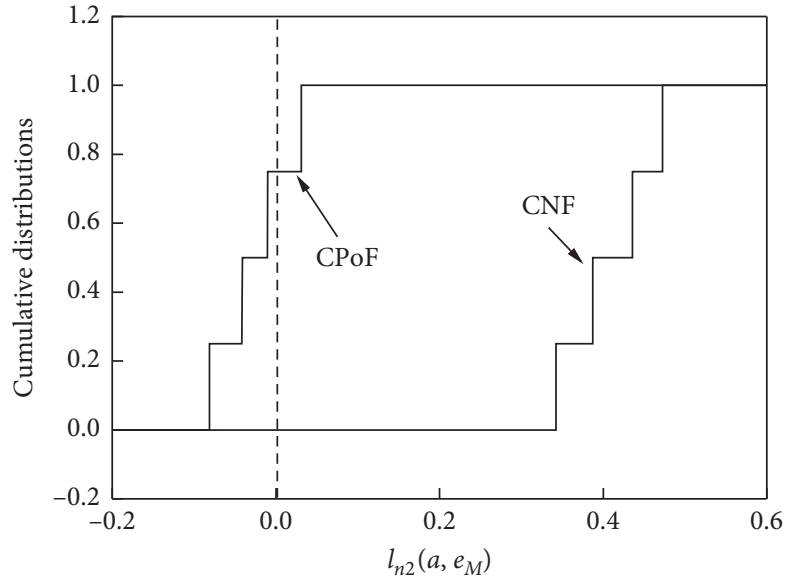

(b)

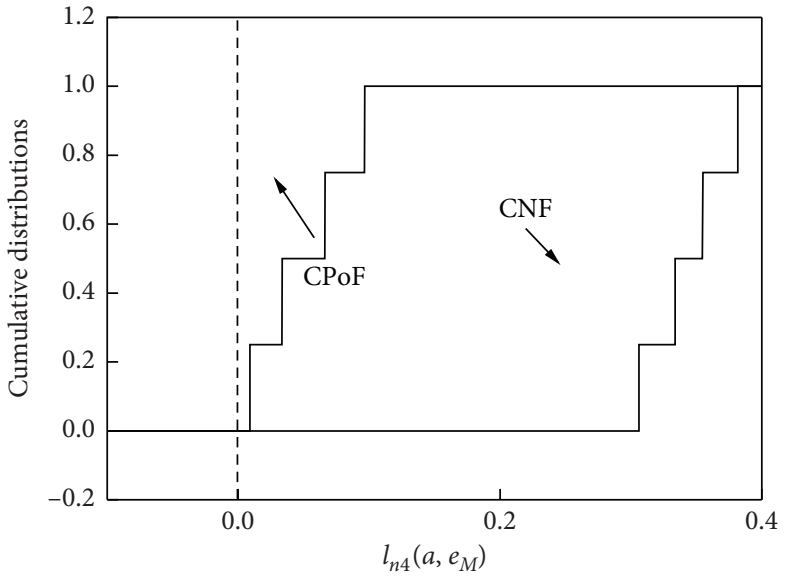

(d)

FIGURE 10: Uncertainty connected with normalized margins $l_{n k}\left(a, e_{M}\right), k=1,2,3,4$, defined in equation (33) characterized by possibility summarized with CPoF and CNF: (a) $l_{n 1}\left(a, e_{M}\right)$ for $l_{n 1}=1.55$, (b) $l_{n 2}\left(a, e_{M}\right)$ for $l_{n 2}=1.75$, (c) $l_{n 3}\left(a, e_{M}\right)$ for $l_{n 3}=2.4$, and (d) $l_{n 4}\left(a, e_{M}\right)$ for $l_{n 4}=2.6$.

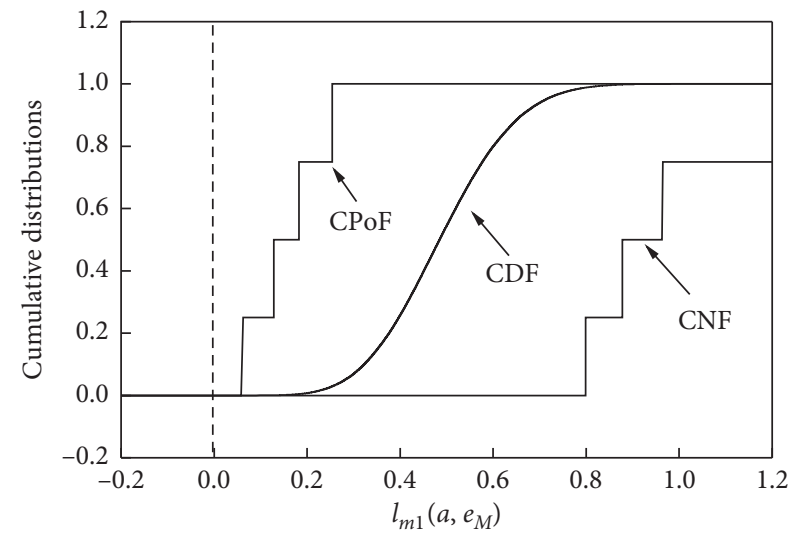

(a)

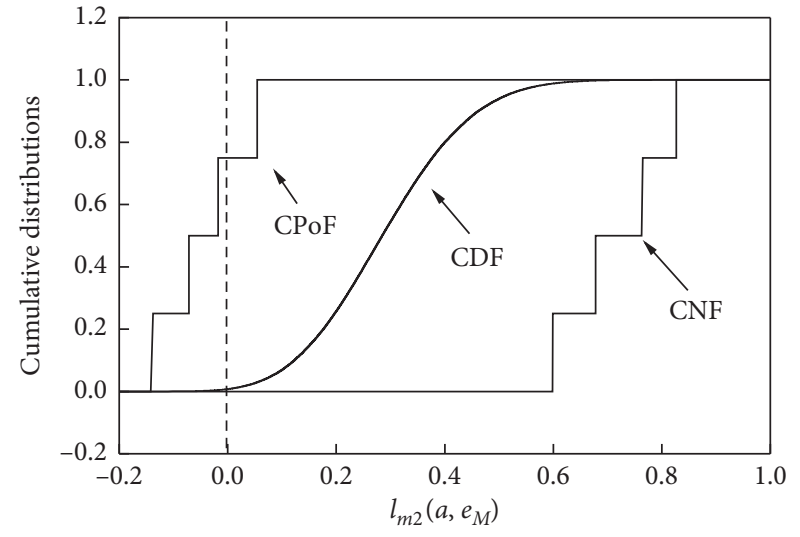

(b)

Figure 11: Continued. 


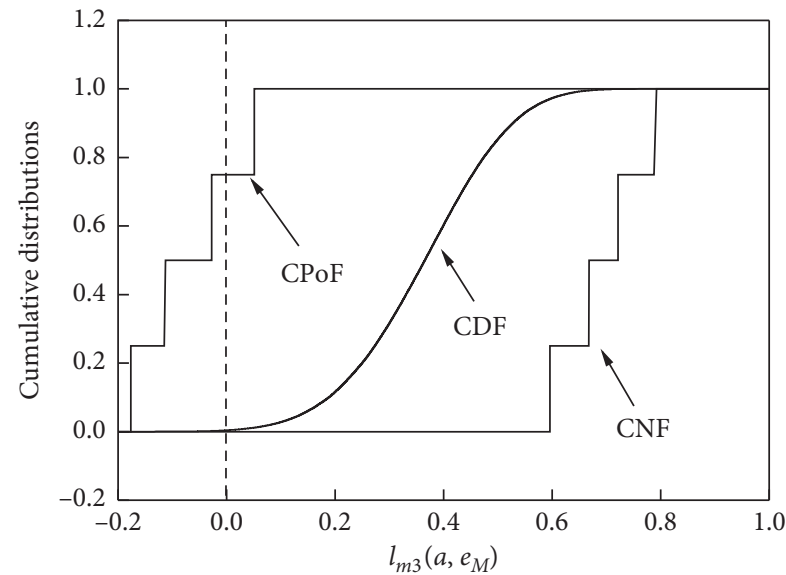

(c)

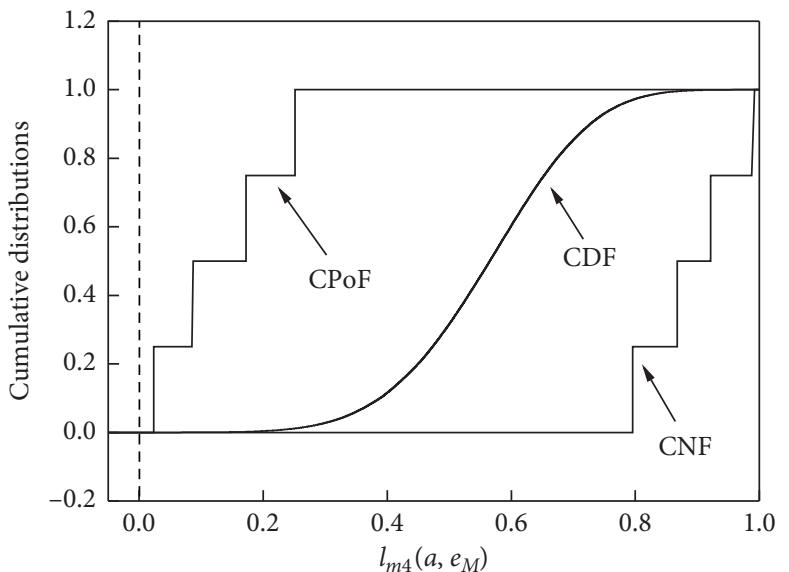

(d)

FIGURE 11: Uncertainty connected with margins $l_{m k}\left(a, e_{M}\right), k=1,2,3,4$, characterized by possibility summarized with CPoF and CNF and by probability summarized with CDF: (a) $l_{m 1}\left(a, e_{M}\right)$ for $l_{m 1}=1.55$, (b) $l_{m 2}\left(a, e_{M}\right)$ for $l_{n 2}=1.75$, (c) $l_{m 3}\left(a, e_{M}\right)$ for $l_{m 3}=2.4$, and (d) $l_{m 4}\left(a, e_{M}\right)$ for $l_{m 4}=2.6$.

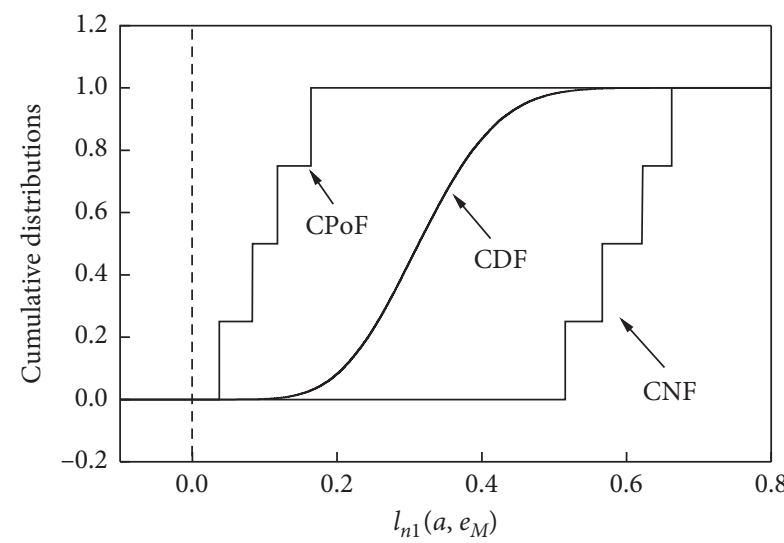

(a)

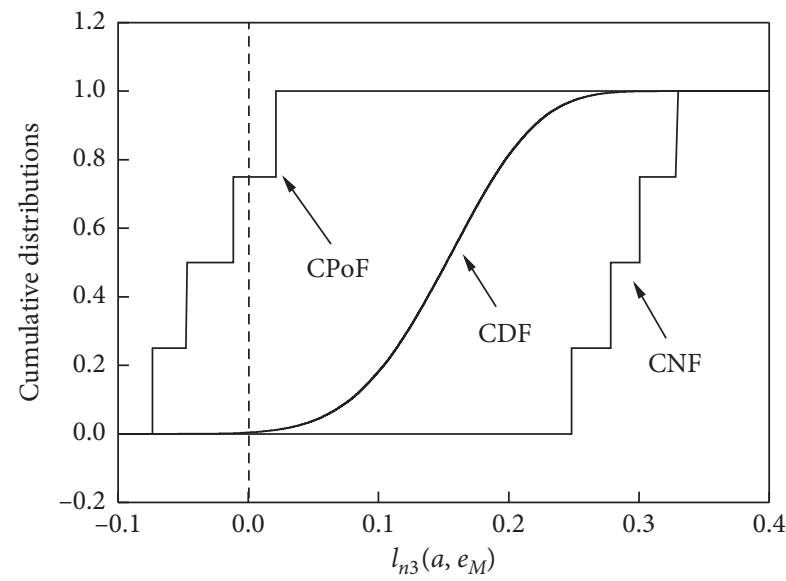

(c)

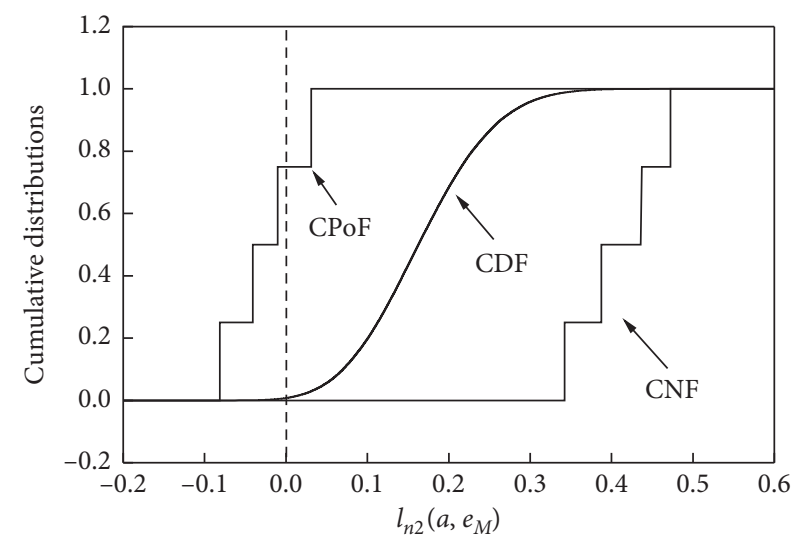

(b)

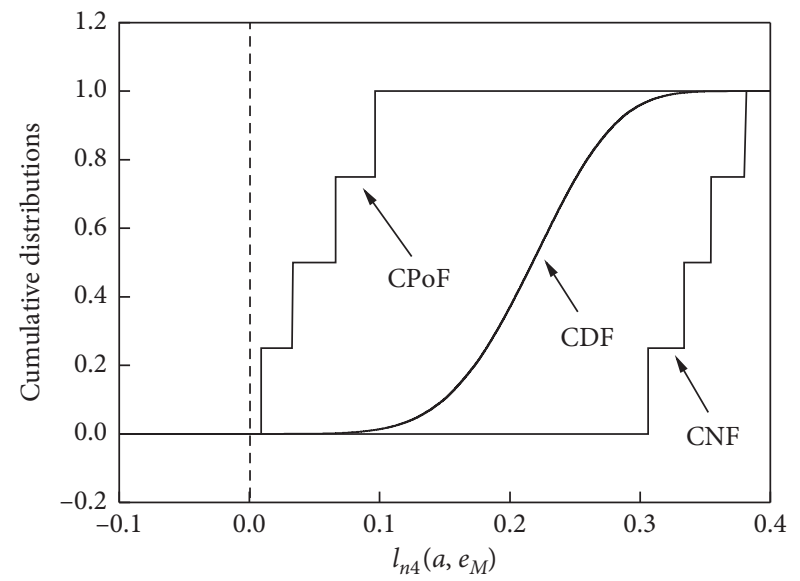

(d)

FIGURE 12: Uncertainty connected with normalized margins $l_{n k}\left(a, e_{M}\right), k=1,2,3,4$, characterized by possibility summarized with CPoF and CNF and by probability summarized with CDF: (a) $l_{n 1}\left(a, e_{M}\right)$ for $l_{n 1}=1.55$, (b) $l_{n 2}\left(a, e_{M}\right)$ for $l_{n 2}=1.75$, (c) $l_{n 3}\left(a, e_{M}\right)$ for $l_{n 3}=2.4$, and (d) $l_{n 4}\left(a, e_{M}\right)$ for $l_{n 4}=2.6$. 
are two measures from the possibility theory. The fundamental difference in the two structures is that the possibilistic one is based on the families of nested sets and the probabilistic one is based on singletons. Furthermore, the difference in distributions is described as follows: the largest values are required to be 1 in the possibility theory but to add to 1 in the probability theory. Each theory is suitable for different models in different mathematical properties.

\section{Summary and Prospects}

With adequate uncertainty representation, the risk of water inrush in karst tunnels can be suitably assessed. The important decisions will be based on the analysis according to the implications and effects of uncertainty, which must be represented by an appropriate form. QMU may be an appropriate method for the representation of uncertainty with possibility.

In addition, possibility is an appropriate mathematical structure that can characterize uncertainty. Risk analyses for water inrush in karst tunnels include the representations of both aleatory and epistemic uncertainties.

Both aleatory and epistemic uncertainties were represented by the probability theory. However, many other mathematical structures have been presented instead of probability theory; for example, interval analysis, evidence theory, and possibility theory. In this paper, possibility for the representation of aleatory uncertainty and epistemic uncertainty is described, and then, it is demonstrated under the conditions of QMU. Through an example, it is illustrated that the possibility theory is better than the probability theory in the representation of epistemic uncertainty for risk analyses of water inrush in karst tunnels.

In particular, if the distributions of the parameters are known and more information is given, the probability theory may be a good mathematical structure for the representation of uncertainty. However, only little information of water inrush in the karst tunnel is available during construction. Thus, the possibility theory is a better mathematical structure than the probability theory because it (1) allows more uncertainty information than interval analysis, (2) permits the assumption of less detailed information than the probability theory, and (3) describes a differentiation between the amount of convenient knowledge supporting a proposition that water inrush of the karst tunnel will occur and the amount of convenient knowledge that does not refute the proposal.

\section{Data Availability}

The data used to support the findings of this study are included within the article.

\section{Conflicts of Interest}

The authors declare no competing financial interest.

\section{Acknowledgments}

The authors would like to thank Editage for English language editing. This study was funded by the Key Project of Chinese National Programs for Fundamental Research and Development (2017YFC0804702) and Scientific Research Project of Jiangsu Geology and Mineral Exploration Bureau (2016KY-1 and 2018-KY-1).

\section{References}

[1] T. Aven, "Perspectives on risk in a decision-making contextreview and discussion," Safety Science, vol. 47, no. 6, pp. 798-806, 2009.

[2] S.-C. Li, Z.-Q. Zhou, L.-P. Li, Z.-H. Xu, Q.-Q. Zhang, and S.-S. Shi, "Risk assessment of water inrush in karst tunnels based on attribute synthetic evaluation system," Tunnelling and Underground Space Technology, vol. 38, pp. 50-58, 2013.

[3] A. Jurado, F. De Gaspari, D. Bolster, X. Sánchez-Vila, D. Fernàndez-Garcia, and D. M. Tartakovsky, "Probabilistic analysis of groundwater-related risks at subsurface excavation sites," Engineering Geology, vol. 125, no. 1, pp. 35-44, 2012.

[4] Y. Wang, W. Yang, M. Li, and X. Liu, "Risk assessment of floor water inrush in coal mines based on secondary fuzzy comprehensive evaluation," International Journal of Rock Mechanics and Mining Sciences, vol. 52, no. 6, pp. 50-55, 2012.

[5] J. Li and L. Chang, "Study on karst water inrush risk assessment model based on karst distribution analysis," Tunnel Construction, vol. 35, no. 8, pp. 792-800, 2015.

[6] Z. Yang and C. Ma, "Risk prediction of water inrush of karst tunnels based on bp neural network," Tunnel Construction, vol. 36, no. 11, pp. 1337-1342, 2016.

[7] P. L. Li, S. C. Li, and Q. S. Zhang, "Study of mechanism of water inrush induced by hydraulic fracturing in karst tunnels," Rock \& Soil Mechanic, vol. 31, no. 2, pp. 523-528, 2010.

[8] G. Fernandez and J. Moon, "Excavation-induced hydraulic conductivity reduction around a tunnel-part 1: guideline for estimate of ground water inflow rate," Tunnelling and Underground Space Technology, vol. 25, no. 5, pp. 560-566, 2010.

[9] D. Kolymbas and P. Wagner, "Groundwater ingress to tunnels-The exact analytical solution," Tunnelling and Underground Space Technology, vol. 22, no. 1, pp. 23-27, 2007.

[10] J.-S. Moon, "Representativeness of jointed rock mass hydraulic conductivity obtained from packer tests for tunnel inflow rate estimate," International Journal of Rock Mechanics and Mining Sciences, vol. 48, no. 5, pp. 836-844, 2011.

[11] Y. Hao, X. Rong, H. Lu, Z. Xiong, and X. Dong, "Quantification of margins and uncertainties for the risk of water inrush in a karst tunnel: representations of epistemic uncertainty with probability," Arabian Journal for Science and Engineering, vol. 43, no. 4, pp. 1627-1640, 2018.

[12] H. Prade and D. D.C. Testemale, Théorie des Possibilités: Applications à la Représentation des Connaissances en Informatique, Bloomington, IN, USA, 1985.

[13] J. C. Helton and J. D. Johnson, "Quantification of margins and uncertainties: alternative representations ofepistemicuncertainty," Reliability Engineering and System Safety, vol. 96, pp. 1034-1052, 2011.

[14] J. C. Helton, W. L. Oberkampf, and J. D. Johnson, "Competingfailure risk analysis using evidence theory," RiskAnalysis, vol. 25, no. 25, pp. 973-995, 2005.

[15] K. Thomas, "About assessing and evaluating uncertain inferences within the theory of evidence," Decision Support Systems, vol. 4, no. 4, pp. 433-439, 1988. 
[16] C. JoslynandJ and C. Helton, "Bounds on belief and plausibility of functionally propagated random sets," in Proceedings of the 2002 Annual Meeting of the North American Fuzzy Information Processing Society Proceedings. NAFIPS-FLINT 2002 (Cat. No. 02TH8622), pp. 412-417, IEEE, New Orleans, LA, USA, June 2002.

[17] J. Kohlas, "Modeling uncertainty with belief functions in numerical models," European Journal of Operational Research, vol. 40, no. 3, pp. 377-388, 1989.

[18] V. Y. Kreinovich, A. Bernat, W. Borrett, Y. Mariscal, and E. Villa, "Monte-Carlo methods make Dempster-Shafer formalism feasible," Advances in the Dempster-Shafer Theory of Evidence, pp. 175-191, NASA, Washington, DC, USA, 1994.

[19] National Research Council (US), "Improving risk communication," Department of Health' Risk Decision \& Policy, vol. 6, no. 2, pp. 159-165, 1989.

[20] National Research Council (US), Science and Judgment in Risk Assessment, National Research Council (US), Washington, DC, USA, 1994.

[21] L. P. Li, Study on Catastrophe evolution mechanism of Karst water inrush, Ph.D. thesis, Shandong University, Jinan, Shandong, 2009.

[22] L. A. Zadeh, "Fuzzy sets as a basis for a theory of possibility," Fuzzy Sets and Systems, vol. 1, no. 1, pp. 3-28, 1978.

[23] R. Krishnapuram and J. M. Keller, "A possibilistic approach to clustering," IEEE Transactions on Fuzzy Systems, vol. 1, no. 2, pp. 98-110, 1993.

[24] D. Dubois, "Possibility theory and statistical reasoning," Computational Statistics \& Data Analysis, vol. 51, no. 1, pp. 47-69, 2001.

[25] C. Baudrit and D. Dubois, "Practical representations of incomplete probabilistic knowledge," Computational Statistics \& Data Analysis, vol. 51, no. 1, pp. 86-108, 2006.

[26] C. Baudrit, D. Dubois, and D. Guyonnet, "Joint propagation and exploitation of probabilistic and possibilistic information in risk assessment," IEEE Transactions on Fuzzy Systems, vol. 14, no. 5, pp. 593-608, 2006.

[27] C. Baudrit, D. Dubois, and N. Perrot, "Representing parametric probabilistic models tainted with imprecision," Fuzzy Sets and Systems, vol. 159, no. 15, pp. 1913-1928, 2008.

[28] G. J. Klirand J and M. Wierman, Uncertainty-based Information, Physica-Verlag-Springer, Berlin, Germany, 1999.

[29] A. Kaufmann and M. M. Gupta, "Introduction to fuzzy arithmetic: theory and applications," Harvard Law Review, vol. 43, no. 43, pp. 2744-2751, 2012.

[30] R. Giles, "Foundations for a theory of possibility," Fuzzy Information \& Decision Processes, vol. 43, pp. 183-195, 1982.

[31] A. Mofarrah and T. Husain, "Fuzzy based health risk assessment of heavy metals introduced into the marine environment," Water Quality, Exposure and Health, vol. 3, no. 1, pp. 25-36, 2011.

[32] M. Kantardzic, Fuzzy Sets and Fuzzy Logic, pp. 414-446, Prentice Hall PTR, Upper Saddle River, NJ, USA, 1995.

[33] C. Baudrit, D. Dubois, and D. Guyonnet, "Joint propagation and exploitation of probabilistic and possibilisticinformation in risk assessment," IEEE Transactions on Fuzzy Systems, vol. 14 , no. 5 , pp. 593-608. 